01.4;02.1

\title{
Влияние концентрации вакансий на скорость миграции границ наклона в никеле: молекулярно-динамическое моделирование
}

\author{
(ㄷ Г.М. Полетаев ${ }^{1}$, Р.Ю. Ракитин ${ }^{2}$ \\ ${ }^{1}$ Алтайский государственный технический университет им. И.И. Ползунова, Барнаул, Россия \\ ${ }^{2}$ Алтайский государственный университет, Барнаул, Россия \\ E-mail: gmpoletaev@mail.ru
}

Поступило в Редакцию 8 декабря 2020г.

В окончательной редакции 10 января 2021 r.

Принято к публикации 12 января 2021 r.

\begin{abstract}
Методом молекулярной динамики проведено исследование влияния концентрации вакансий на скорость миграции большеугловых границ наклона с осями разориентации $\langle 111\rangle$ и $\langle 100\rangle$ в никеле. Показано, что зависимость скорости миграции границ зерен от концентрации вакансий немонотонна и имеет максимум при концентрации введенных на начальном этапе вакансий около $1 \%$. При дальнейшем повышении концентрации, особенно свыше 4\%, скорость миграции рассматриваемых границ падает в результате торможения границ прикрепляющимися к ней малоподвижными вакансионными кластерами, которые граница уже не может сорбировать, как в случае сравнительно малых кластеров.
\end{abstract}

Ключевые слова: молекулярная динамика, миграция границы зерен, вакансия, свободный объем.

DOI: 10.21883/PJTF.2021.08.50850.18648

Миграция границ зерен в поликристаллах имеет определяющее значение в процессах рекристаллизации, роста зерен, во многих фазовых превращениях. Подвижность границ критически влияет на стабильность микроструктуры ультрамелкозернистых, в том числе нанокристаллических, материалов [1-3]. Известно, что скорость миграции границы зерен зависит от ее ориентации, температуры, деформации, наличия примесей или собственных дефектов кристаллической структуры $[1,2]$. Последние в большинстве случаев тормозят миграцию границ [1-5]. Исключением являются вакансии, которые, согласно, например, косвенным экспериментальным данным [6,7] и результатам $a b$ initio моделирования [8,9], не только не препятствуют миграции, но и приводят к ее ускорению. Вместе с тем в обоих случаях имеются факторы, усложняющие однозначную интерпретацию влияния концентрации вакансий на подвижность границ. В случае экспериментальных исследований это, как правило, сложность выделения влияния только вакансий наряду с другими дефектами и локальным разогревом, возникающим вследствие внешних воздействий (облучения частицами, деформации), создаваемых для повышения концентрации вакансий. Другой проблемой, общей для экспериментальных и $a b$ initio методов, является сложность определения фактического значения концентрации вакансий при высоких ее значениях из-за процессов абсорбции вакансий самими границами и объединения вакансий друг с другом с образованием вакансионных кластеров. С другой стороны, в $[10,11]$ делается вывод о конечной сорбционной способности границ зерен по отношению к вакансиям и свободному объему, вследствие чего, в частности, сокращение площади границы в результате миграции сопровождается эмиссией избыточного свободного объема в виде вакансий обратно в кристалл, что в свою очередь снижает миграционную подвижность границы. Оба указанных выше явления, по всей видимости, должны приводить к немонотонной зависимости скорости миграции границ зерен от концентрации вакансий.

Настоящая работа посвящена исследованию влияния свободного объема на скорость миграции большеугловых границ наклона с осями разориентации $\langle 111\rangle$ и $\langle 100\rangle$ в никеле методом молекулярной динамики. Преимуществом модели является то, что при постоянной температуре объем расчетной ячейки оставался постоянным, как и вводимый в расчетную ячейку на начальном этапе дополнительный свободный объем, несмотря на процессы абсорбции вакансий границей и образование вакансионных кластеров. Границы наклона $\langle 111\rangle$ и $\langle 100\rangle$ были выбраны в связи с их сравнительно высокой подвижностью, позволяющей проводить измерения скорости их миграции в молекулярно-динамических экспериментах [12,13]. При этом наибольшую подвижность, как известно, имеют большеугловые границы. Для рассмотрения был взят угол разориентации $30^{\circ}$. В качестве моделируемого металла был выбран никель как один из типичных и хорошо изученных ГЦК-металлов.

За основу модели была взята методика исследования миграции границы зерен наклона, предложенная и развитая в работах $[1,4,14]$. В этом случае создается четко аттестованная граница в форме петли или арки, как на рис. 1 (показана темной штриховой линией). Сила натяжения границы, которая подобно поверхностному натяжению возникает вследствие стремления границы минимизировать свою энергию, является причиной направленного перемещения границы в сторону уменьше- 


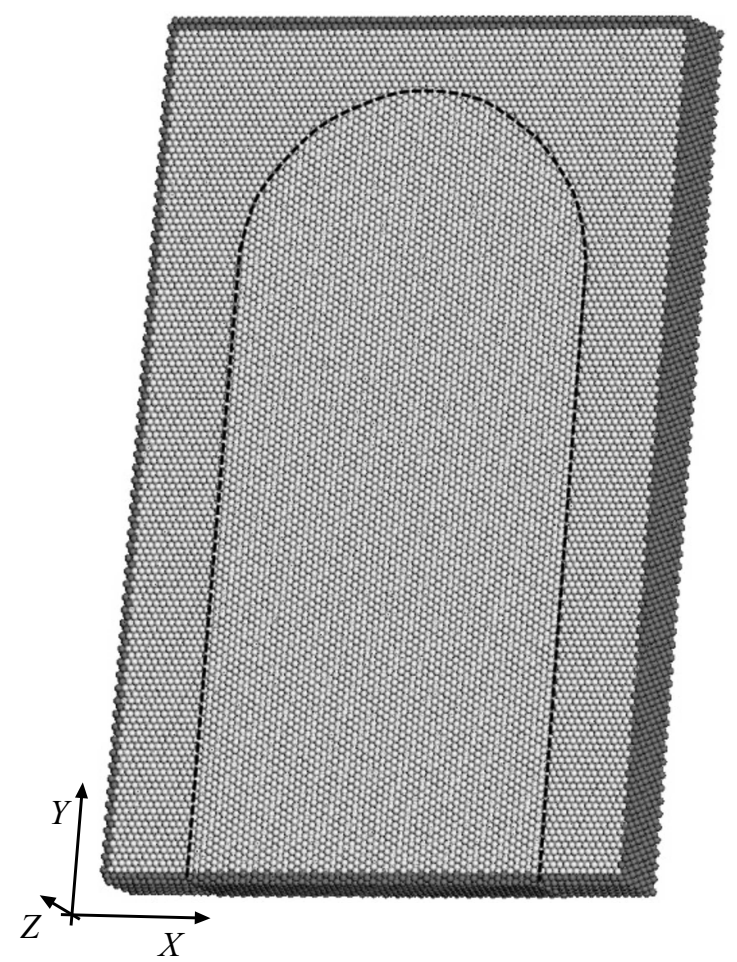

Рис. 1. Расчетная ячейка для моделирования миграции границы наклона $\langle 111\rangle 30^{\circ}$. Темно-серые атомы на краю расчетной ячейки в процессе компьютерного эксперимента оставались неподвижными (жесткие граничные условия).

ния ее площади. Сила, провоцирующая миграцию, зависит от угла разориентации (с ростом угла разориентации растет, как правило, и энергия границы) и кривизны границы. В нашей модели граница создавалась с относительно высокой кривизной для того, чтобы скорость миграции была достаточно высокой, чтобы ее можно было измерить методом молекулярной динамики. Вместе с тем она не должна быть и слишком большой, чтобы не приводить к аномально высоким скоростям миграции, провоцирующим, как, например, в [14], интенсивное испускание вакансий быстро мигрирующей границей. Сила и скорость миграции при постоянной температуре оставались в рассматриваемой модели постоянными в течение почти всего времени движения границы, плавно уменьшаясь к концу компьютерного эксперимента.

Расчетная ячейка содержала примерно 120000 атомов и имела высоту $28.5 \mathrm{~nm}$, ширину $19.0 \mathrm{~nm}$ и толщину $2.4 \mathrm{~nm}$. Вдоль оси $Z$ (рис. 1) имитировалось бесконечное повторение структуры, т.е. были наложены периодические граничные условия. На краю расчетной ячейки границы зерен должны быть зафиксированы, что подразумевает сохранение ориентации кристаллической решетки двух разных зерен на границе ячейки. В связи с этим по осям $X$ и $Y$ границы ячейки были жестко закреплены для фиксации заданной разориентации зерен: атомы, окрашенные в темно-серый цвет на рис. 1, оставались неподвижными в процессе компьютерного эксперимента.

Для описания взаимодействий атомов никеля друг с другом использовались многочастичные потенциалы Клери-Росато [15], построенные в приближении сильной связи. Потенциалы данного типа неоднократно использовались в молекулярно-динамических моделях и прошли апробацию по большому числу характеристик [16,17]. Опыт их применения показывает, что с их помощью удается описать разнообразные свойства металлов и сплавов. Шаг интегрирования по времени в методе молекулярной динамики был равен $2 \mathrm{fs}$. Температура в модели задавалась через начальные скорости атомов согласно распределению Максвелла-Больцмана, при этом учитывалось тепловое расширение расчетных блоков. Для сохранения температуры постоянной в процессе моделирования использовался термостат Нозе-Гувера. Моделирование миграции границ проводилось при $1700 \mathrm{~K}$. Выбор температуры почти равной температуре плавления никеля обусловлен тем, что при ней скорость миграции границ наибольшая. Концентрация вакансий, вводимых в расчетную ячейку, на начальном этапе моделирования варьировалась от 0 до 7\%. Последнее значение на два порядка превышает равновесную концентрацию вакансий, но вместе с тем это позволяет рассмотреть влияние вакансионных кластеров, возникающих, например, при радиационном повреждении, на подвижность границ. Суммарный дополнительный свободный объем в расчетной ячейке, т.е. объем свободного пространства свыше того, который соответствует идеальному кристаллу, складывался таким образом из вакансионного и свободного объема, содержащегося изначально в границе зерен $(1.2 \%$ от всего объема расчетной ячейки).

На рис. 2 изображены зависимости скорости миграции рассматриваемых границ зерен при температуре $1700 \mathrm{~K}$ от концентрации вакансий, введенных на начальном

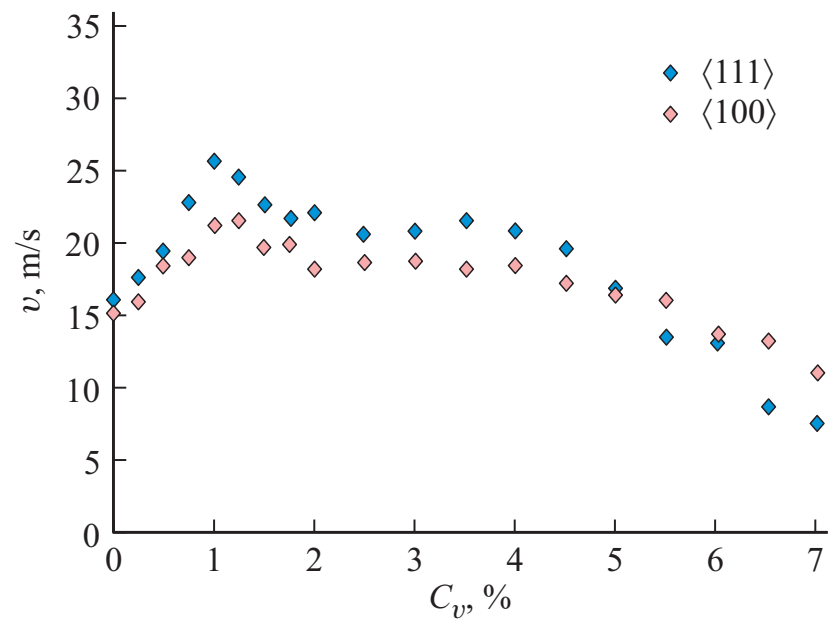

Рис. 2. Зависимости скорости миграции границ наклона $\langle 111\rangle$ и $\langle 100\rangle$ с углом разориентации $30^{\circ}$ при температуре $1700 \mathrm{~K}$ от начальной концентрации вакансий в расчетной ячейке. 

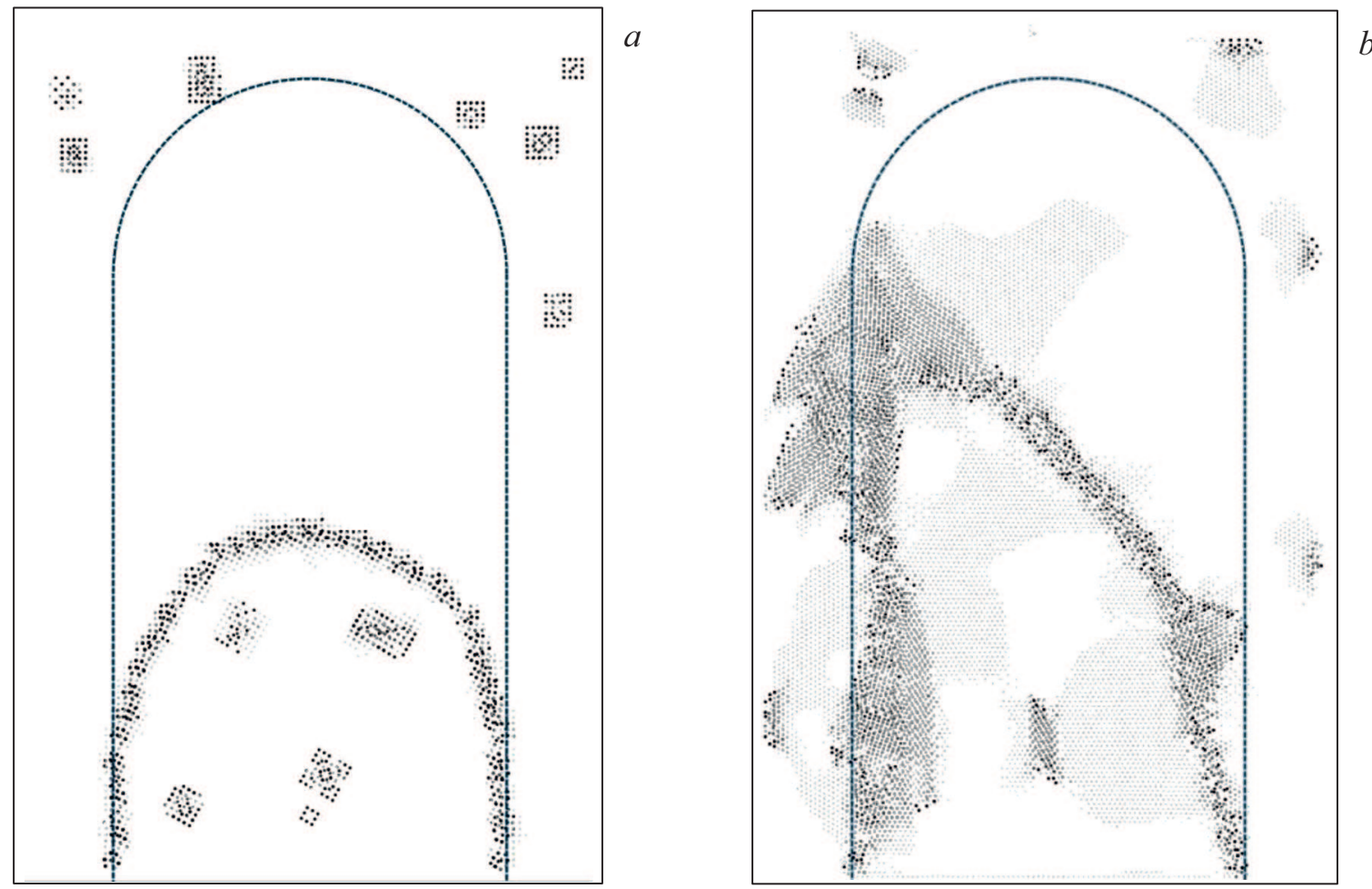

Рис. 3. Примеры распределения свободного объема в расчетной ячейке после моделирования миграции границы. $a-$ поглощение вакансий и вакансионных кластеров границей $\langle 100\rangle 30^{\circ}$ в результате ее миграции при начальной концентрации вакансий $0.5 \%$ в течение $700 \mathrm{ps} ; b$ - задерживание миграции границы $\langle 111\rangle 30^{\circ}$ вакансионными кластерами (начальная концентрация вакансий $5.5 \%$, время с начала моделирования 800 ps). Изображения получены после охлаждения структуры расчетной ячейки.

этапе компьютерного эксперимента. Скорость миграции оценивалась по смещению верхней части границы в форме полупетли (рис. 1) вдоль оси $Y$. Столь высокие значения скорости (от 7 до $26 \mathrm{~m} / \mathrm{s}$ ) характерны для молекулярно-динамического моделирования миграции границ зерен и достигнуты в данном случае благодаря сочетанию трех факторов: чрезвычайно высокой изначальной кривизны границы, высокой температуры, близкой к температуре плавления, и высокой подвижности рассматриваемых границ.

Как видно, при сравнительно небольших концентрациях вакансий (примерно до 1\%) с ростом концентрации повышается и скорость миграции границ, что согласуется с косвенными экспериментальными данными [6,7]. Увеличение скорости миграции, очевидно, связано с повышением диффузионной подвижности атомов на границе зерен с ростом свободного объема. Как было показано, например, в [13], миграция границ наклона осуществляется преимущественно путем согласованных (кооперативных) смещений атомов в зерне, в сторону которого происходит миграция. Такие смещения, по всей видимости, облегчены при наличии дополнительного свободного пространства - свободного объема, на что также косвенно указывает, например, влияние гидростатического сжатия на скорость миграции [18].

При концентрациях вакансий до $3 \%$ характерным было явление „очищения“ кристалла движущейся гра- ницей зерен. В процессе миграции граница поглощала встречающиеся на ее пути вакансии и вакансионные кластеры. На рис. 3, a с помощью визуализатора среднего расстояния до ближайших атомов показан пример подобного явления при миграции границы $\langle 100\rangle 30^{\circ}$ при начальной концентрации вакансий $0.5 \%$ в течение 700 ps. Данный визуализатор дает представление о распределении свободного объема. Для каждого атома проводился расчет среднего расстояния до ближайших атомов. Если среднее расстояние незначительно отличалось от расстояния, соответствующего идеальному кристаллу, атом не изображался. В противном случае атом закрашивался в тот или иной оттенок серого (чем темнее, тем больше свободного объема вблизи данного атома).

С дальнейшим ростом концентрации вакансий более $1 \%$ скорость миграции границ $\langle 111\rangle$ и $\langle 100\rangle$, как видно из рис. 2, напротив, снижается, а при концентрации выше 4\% начинает падать еще сильнее. Основной причиной этого является торможение границ прикрепляющимися к ней малоподвижными вакансионными кластерами, которые при данных концентрациях уже достаточно большие, и граница не способна их полностью сорбировать. Сравнительно большие вакансионные кластеры в этом случае начинают работать как большинство дефектов или инородных частиц, имеющих положительную энергию связи с границей зерен и одновременно слабую диффузионную подвижность, что вместе приводит к 
снижению миграционной подвижности границы [1-3]. На рис. 3, $b$ изображен пример задержки границы подобным вакансионным кластером.

Другой причиной снижения скорости миграции границ является рассмотренное в работах $[10,11]$ уменьшение движущей силы из-за конечной сорбционной способности по отношению к вакансиям. При чрезмерно большой величине сорбированного границей дополнительного свободного объема наступает момент, когда сокращение ее площади приводит к частичной эмиссии свободного объема в виде вакансий обратно в кристалл. Поверхностное натяжение границы зерен и соответственно движущая сила миграции в таком случае снижаются.

Таким образом, в работе с помощью молекулярно-динамического моделирования на примере границ наклона $\langle 111\rangle$ и $\langle 100\rangle 30^{\circ}$ в никеле показано, что зависимость скорости миграции границ зерен от свободного объема немонотонна и имеет максимум вблизи 1\% введенных на начальном этапе вакансий. При концентрациях вакансий до 3\% характерным было явление „очищения“ кристалла движущейся границей зерен: в процессе миграции граница поглощала встречающиеся на ее пути вакансии и вакансионные кластеры. При дальнейшем повышении концентрации, особенно свыше 4\%, скорость миграции рассматриваемых границ падала в результате торможения границ прикрепляющимися к ней малоподвижными вакансионными кластерами, которые граница уже не могла сорбировать, как в случае сравнительно малых кластеров.

\section{Конфликт интересов}

Авторы заявляют, что у них нет конфликта интересов.

\section{Список литературы}

[1] G. Gottstein, L.S. Shvindlerman, Grain boundary migration in metals: thermodynamics, kinetics, applications, 2nd ed. (CRC Press, Boca Raton, 2009).

[2] О.А. Кайбышев, Р.З. Валиев, Гранищы зерен и свойства металлов (Металлургия, М., 1987).

[3] Q. Zhu, S.C. Zhao, C. Deng, X.H. An, K.X. Song, S.X. Mao, J.W. Wang, Acta Mater., 199, 42 (2020). DOI: 10.1016/j.actamat.2020.08.021

[4] G. Gottstein, D.A. Molodov, L.S. Shvindlerman, Interface Sci., 6 (1-2), 7 (1998). DOI: 10.1023/A:1008641617937

[5] L.-L. Niu, Q. Peng, F. Gao, Zh. Chen, Y. Zhang, G.-H. Lu, J. Nucl. Mater., 512, 246 (2018). DOI: $10.1016 /$ j.jnucmat.2018.10.014

[6] F. Haessner, J. de Phys. Coll., 36 (C4), 345 (1975). DOI: $10.1051 /$ jphyscol:1975435

[7] H. Takahashi, N. Hashimoto, Mater. Trans., 34 (11), 1027 (1993). DOI: 10.2320/matertrans1989.34.1027

[8] G. Lu, N. Kioussis, Phys. Rev. B, 64 (2), 024101 (2001). DOI: 10.1103/PhysRevB.64.024101

[9] P. Ballo, N. Kioussis, G. Lu, Phys. Rev. B, 64 (2), 024104 (2001). DOI: 10.1103/PhysRevB.64.024104
[10] Y. Estrin, G. Gottstein, E. Rabkin, L.S. Shvindlerman, Acta Mater., 49 (4), 673 (2001). DOI: $10.1016 / \mathrm{S} 1359-6454(00) 00344-\mathrm{X}$

[11] V.G. Sursaeva, G. Gottstein, L.S. Shvindlerman, Scripta Mater., 116, 91 (2016). DOI: 10.1016/j.scriptamat.2016.01.021

[12] Y. Huang, F.J. Humphreys, Mater. Chem. Phys., 132 (1), 166 (2012). DOI: 10.1016/j.matchemphys.2011.11.018

[13] Г.М. Полетаев, И.В. Зоря, М.Д. Старостенков, Р.Ю. Ракитин, П.Я. Табаков, ЖЭТФ, 155 (1), 96 (2019). DOI: 10.1134/S0044451019010073 [Пер. версия: $10.1134 / \mathrm{S} 1063776118120087]$.

[14] С.Г. Протасова, В.Г. Сурсаева, Л.С. Швиндлерман, ФТТ, 45 (8), 1402 (2003). [Пер. версия: 10.1134/1.1602881].

[15] F. Cleri, V. Rosato, Phys. Rev. B, 48 (1), 22 (1993). DOI: 10.1103/PhysRevB.48.22

[16] G.M. Poletaev, I.V. Zorya, R.Y. Rakitin, M.A. Iliina, Mater. Phys. Mech., 42 (4), 380 (2019). DOI: 10.18720/MPM.4242019_2

[17] Г.М. Полетаев, И.В. Зоря, Письма в ЖТФ, 46 (12), 6 (2020). DOI: 10.21883/PJTF.2020.12.49518.18279 [Пер. версия: 10.1134/S1063785020060231].

[18] D.A. Molodov, B.B. Straumal, L.S. Shvindlerman, Scripta Met., 18 (3), 207 (1984). DOI: $10.1016 / 0036-9748(84) 90509-\mathrm{X}$ 